\title{
Adjustment and transformation strategies of ItalPoS Permanent GNSS Network
}

\author{
Cristina Castagnetti $\left({ }^{1}\right)$, Giuseppe Casula $\left({ }^{2}\right)$, Marco Dubbini $\left({ }^{1}\right)$ and Alessandro Capra $\left({ }^{1}\right)$ \\ (1) DIMeC - Dipartimento di Ingegneria Meccanica e Civile, \\ Università degli Studi di Modena e Reggio Emilia, Modena, Italy \\ $\left(^{2}\right)$ INGV, Istituto Nazionale di Geofisica e Vulcanologia, Bologna, Italy
}

\begin{abstract}
A private network of 133 Global Navigation Satellite Systems (GNSS) permanent sites, named ItalPoS (Italian Positioning Service) and uniformly covering the entire Italian territory, was established in April 2006 by the Italian Division of Leica Geosystems S.p.A. This network also involves several GPS stations of the INGV (Italian National Institute of Geophysics and Volcanology) RING (Real-time Integrated National Gps) network and GPS stations from other public and private bodies. Indeed Leica Geosystems contributed with its experience and its products to these networks which currently fulfil the national coverage where there are no ItalPoS stations. For instance, the purpose of the ItalPoS network is mainly GNSS data distribution in terms of real time corrections and RINEX files for the post-processing.

In order to compute and distribute to private and scientific users a set of adjusted coordinates of the ItalPoS network in the IGS05 and ETRF89-2000 reference frames, a dataset of RINEX of about 120 GPS permanent sites was created and processed, using the distributed sessions approach, by means of Gamit/Globk 10.3 GPS data processor, also using some SOPAC (Scripps Orbits and Permanent Array Center) and EUREF (European Permanent Network) solutions to improve the reference frame definition and the internal constraints.

This paper describes all the strategies adopted to estimate coordinates and velocities of the ItalPoS Network in IGS05 and in ETRF89-2000 reference frames in the whole Italian territory.
\end{abstract}

Key words GNSS Permanent Network - Globk Adjustment Strategies - Boucher-Altamimi transformation

\section{Introduction}

Nowadays a GNSS (Global Navigation Satellite Systems) permanent network is a strong instrument for people working on geo-

Mailing address: Dr. Cristina Castagnetti, DIMeC Dipartimento di Ingegneria Meccanica e Civile, Università degli Studi di Modena e Reggio Emilia, Via Vignolese 905, 41125 Modena; e-mail: cristina.castagnetti@unimore.it desy, surveying and mapping because it can provide a significant facility for reduction in terms of time and costs during a survey. In addition it provides very important support for studies and researches on crustal deformation or subsidence monitoring, etc.

Recently a new private GNSS permanent network named ItalPoS (Italian Positioning Service) was set up in Italy by Leica Geosystems Italian Division. The network is the first GNSS permanent network at a national scale covering the whole territory. With more than 133 permanent stations uniformly distributed over the Italian peninsula to date, the network, is processed and adjusted in the IGS05 reference frame by the scientific software Gamit/Globk 10.3. To provide users with a complete positioning serv- 
ice, transformation parameters from the network reference frame, IGS05, to the Italian reference frame, IGM95-ETRF89, were estimated. This task is requested by users that need to have results in the official reference frame IGM95 that is the Italian realization of the European ETRF89.

Due to Italian morphology and its differential deformations, and in consideration of error propagation of IGM95 solution, the whole territory was intentionally divided into three macro areas to optimize the transformation estimation. Both processing, adjustment network strategies and transformation parameters estimation will be described below, focusing on the different approaches tested for transformation and on the final choice reasons.

\section{Structure of the ItalPoS permanent network}

About a year ago the Italian division of Leica Geosystems S.p.A built the first private GNSS permanent network at a national scale in Italy. This GNSS Network, named ItalPoS (Italian Positioning Service), consists of a private permanent network uniformly covering the whole Italian territory. The purpose of this network is GNSS data distribution in terms of real time corrections (RTK by means of RTCM 2 and 3 protocols) and RINEX files and METADATA for post-processing. The project was developed in collaboration with many Universities and Research Institutes, which contributed and are still contributing to the network development, improvement and publication. The network structure is made of several high quality GNSS reference stations. Some of them were materialized some years ago and are still managed by the INGV, responsible for the RING permanent network. RING is the largest GNSS monitoring network in terms of extension at national scale (Selvaggi and RING Working Group, 2006). Its purpose is purely scientific and geodetic considering that it does not provide any services for customers. Many stations have Leica Geosystems instruments. Moreover ItalPoS also involves several GPS stations from other public networks such as Puglia, Tuscany and Veneto Regions, Province of Cagliari, Autonomous Province of Bolzano Catasto fondiario e urbano, Autonomous Province of Trento Servizio Catasto, Province of Perugia Collegio Geometri. Indeed Leica Geosystems contributed with its experience and its products to these networks which nowadays fulfil the national coverage where there are no ItalPoS stations.

ItalPoS network, currently composed of more than 133 permanent stations distributed throughout the Italian peninsula (fig. 1), aims to provide customers with a service both in real time and in post-processing. GNSS data are available without charge concerning the real time corrections provided by the nearest station. In addition, ItalPoS provides a service with fee distributing RINEX data for post-processing and real time area corrections. Moreover, more precise and stable results are allowed because the real time corrections can be transmitted not only from the nearest station but from several stations in the clusters the network is suitably split off. The real time service is based on the approach called MAX, MasterAuxiliary (Leica Geosystems, 2005): the data centre provides user with both coordinates and corrections from the nearest station (master) and coordinates and corrections from the other stations (auxiliary) making part of the cluster the user is included in. Thanks to this information, the user receiver develops a spatial errors model, builds the double differences with the master station, makes a correction by means of the errors model and performs a position estimation. In the near future, the user receiver will be able to build a multi-base double differences system for each station in the cluster (without distinction between master and auxiliary stations) and to provide errors and position multi-base estimation.

Thanks to ItalPoS, a user with a single receiver, rover, can access services supplied by a simple GSM (Global System for Mobile Communications) or GPRS (General Packet Radio Service) connection. As a consequence the user can reduce the time and costs of the work and gain satisfaction with every kind of survey without any further difficulties caused by coordinates transformation and geo-referring. At the 


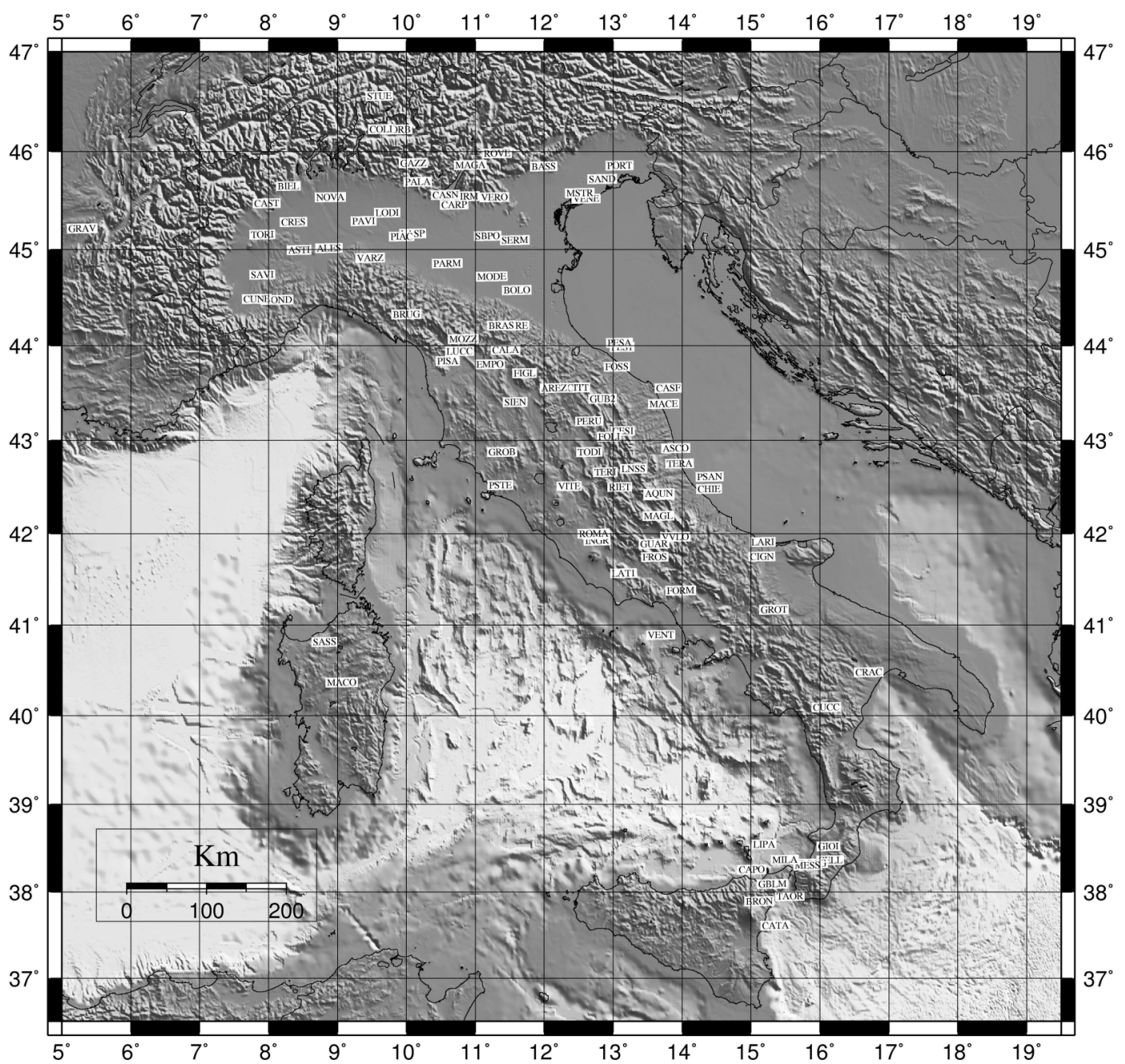

Fig. 1. ItalPoS permanent network: a general map of the network with all the stations involved (both ItalPoS and RING ones).

adjustment and transformation estimation time, the Italian official reference frame was IGM95, the Italian realization of the European reference frame, ETRF89 (Boucher and Altamimi, 1992). As a consequence, ItalPos distributes coordinates in IGM95 reference frame. Currently, a new official reference frame has been introduced by the Istituto Geografico Militare Italiano (IGMI), the ETRF2000. However, a transi- tion period is necessary, so that the results provided in this paper both for stations coordinates and transformation parameters still refer to IGM95-ETRF89.

The network was processed and adjusted in the IGS05 reference frame (Biagi et al., 2008). Transformation parameters were subsequently provided to users to have results in the official Italian reference frame, IGM95-ETRF89. 


\section{GPS data processing and adjustment strategies}

In order to compute a set of coordinates of the described ItalPoS network in the IGS05 reference frame, an array of about 120 GPS PSs maintained by different institutions (INGV, EUREF, ASI, LEICA GEOSYSTEMS and so on) was processed by means of the software Gamit/Globk version 10.3 (Herring et al., 2006a,b) developed at the CfA (Harvard Smitsonian Center of Astrophysics) of MIT (Massachusetts Institute of Technology) and at the SIO (Scripps Institution of Oceanography), University of California (San Diego).

Data were archived on electronic magnetic

Table I. List of the GPS stations used for the ItalPoS network data elaboration (stations for each cluster).

\begin{tabular}{|c|c|c|c|c|}
\hline Cluster Itp1 & Cluster Itp2 & Cluster Itp3 & Cluster Itp4 & Cluster Itp5 \\
\hline ALES & TORI & CITT & PARM & PESA \\
\hline ASCO & VENE & EMPO & TODI & GROT \\
\hline ASTI & SERM & FIGL & VITE & CUCC \\
\hline BIEL & VIGE & FOLI & CHIEL & MILA \\
\hline BOLO & SIRM & GUB2 & BRON & CESI \\
\hline CARP & PINO & LARI & SBPO & CELL \\
\hline CASF & PES1 & LAT1 & GROB & CIGN \\
\hline CASN & PIAC & ROMA & MODE & CRAC \\
\hline CEL1 & DARF & MAGL & GIOI & GBLM \\
\hline COLI & PORT & MESS & TEMP & GUAR \\
\hline CUNE & SAND & PALA & FROS & LNSS \\
\hline FORL & VERO & PERU & CATA & VENT \\
\hline GAZZ & MACE & PISA & LIPA & VLSG \\
\hline LODI & VARZ & RIET & GRAV & VVLO \\
\hline MOD1 & BRUG & PESS & MOZZ & INGR \\
\hline MOND & MACO & SASS & CALA & BASS \\
\hline MORB & CAST & MAGA & LUCC & SIEN \\
\hline NOVA & VERC & TERI & FIRE & CRES \\
\hline PAVI & AQUN & TAOR & STUE & FORM \\
\hline ROVE & AREZ & TERA & LASP & FOSS \\
\hline \multirow[t]{2}{*}{ SAVI } & PSTE & CAPO & SCHR & PSAN \\
\hline & & MSTR & & \\
\hline
\end{tabular}


supports by means of an automatic procedure with the aid of scripts of the Unix bourne shell and FORTRAN appropriately implemented and running on a Intel Quad-Core Workstation, suitably projected and assembled for this task, under Linux OpenSuse 11 Operative System (OS).

A multistep procedure was applied based on the distributed sessions approach (Dong et al., 1998; Serpelloni et al., 2006; Casula et al., 2007): the network was divided into five clusters (see table I); then in the first step the GPS data over one month time span (October-November 2007) were processed by means of Gamit software to obtain five daily observation solutions (Gamit h-files). For the sake of compatibility with the SOPAC clustering we applied the same processing setup used by SOPAC: we used precise IGS orbits and Earth Orientation Parameters (EOP) tightly constrained as indicated in the sestbl. processing file suggested by SOPAC for Gamit 10.3 solution of regional clusters. The ambiguity resolution was performed with the aid of the $L C \_A U T C L N$ procedure which uses the Melbourne-Wübbena wide lane (WL) linear combination to resolve double difference ambiguities. In the second step the solutions of the ItalPoS network clusters were format converted and combined with some of the h-files of the Scripps Orbit and Permanent Array Center (SOPAC 2008, http://www.sopac.edu) regional clusters. A set of unified combined $h$-files was created by means of the glred processor of the Globk package using the robust combination criteria described in Dong et al., 1998; Herring et al., 2006a,b.

At this stage of the analysis a reference frame of the solution had to be defined (stabilisation) by means of the Glorg module of the Globk package simply by minimising the deviations between horizontal positions and velocities of our solutions and those given in an a-priori reference station file (Herring et al., 2006b).

The final step consisted of the stabilisation of the ItalPoS network sites by geo-referring them in the IGS05 reference frame and in the ETRF89-2000 one by means of the known positions and velocities data of a set of reference stations, statistically stable, (called fiducial stations): BOR1, BRUS, CAGL, GLSV, GRAS,
HOFN, JOZE, MATE, METS, NICO, NOT1, NYA1, NYAL, ONSA, POLV, POTS, RABT, RAMO, REYK, SFER, TRAB TRO1 VILL WSRT WTZR ZIMM; as indicated by EUREF for the definition of IGS05 reference frame for data processed after November 2006. The positions and velocities of these fiducial stations which were included in the computation are extracted from EUREF facility combined solutions (Bruyninx, 2004; Kenyeres and Bruyninx 2004).

To account for the consistency of the reference frame definition and stability of our solution we computed by means of the Tform program of the Globk package, for about 30 reference sites, the residuals between the coordinates and velocities estimated in our solutions and the ones computed by EUREF at the same epoch; the results of this operation are shown in table II.

Then a set of adjusted coordinates in the ERTF2000 reference frame at epoch 1989.0 were computed for a time span of one month by means of the Glorg program of Globk and the ETRF2000 values of the fiducial sites coordinates and velocities made available by EUREF facility (see also table VIII).

\section{Reference frame transformation parameters}

To provide users with a complete positioning service, transformation parameters from the network reference frame, IGS05, to the Italian reference frame, IGM95-ETRF89, were estimated. This is requested by those users who need to have results in the official reference frame IGM95.

Thinking about Italian morphology and its differential deformations, and in consideration of error propagation of IGM95 solution, the whole territory was intentionally divided into three macro areas to optimize the transformation estimation. A IGS05-IGM95 transformation was estimated and a parameters set provided to customers for each area. First of all, several sets of parameters were estimated by means of different strategies to test multiple results and make comparisons to find the best so- 
lution. This aims to optimize the transformation and choose a unique set of parameters for each area. The following section compares different approaches and discusses parameters results for each specific area.

The first step was to find the best methodology to estimate the parameters. Two approaches were investigated.

The first is a regional adaptation of the international transformations between ITRF05 (Altamimi et al., 2007) and ETRF89 (Boucher and Altamimi, 2007). In detail the well-established Boucher-Altamimi transformation (in the following $\mathrm{BA}$ ), that is a rigid combination of rotation and translation where the rotation is assigned for Europe and the translation depends on the geodynamic velocity of the site. The adaptation at the interest area (parts of Italy) requires some approximations (IGS05 $\cong \mathrm{ITRF} 05$ and IGM95 $\cong E T R F 89$ ) and the estimation of a mean velocity unique for the area. The Boucher-Altamimi transformation equation at the generic time are reported below:

$$
\begin{aligned}
& X_{E 89}(1989.0)=T+X_{105}(\tau)+(\tau-1989.0) \\
& \dot{R} \cdot X_{105}(\tau)+(1989.0-\tau) \cdot \dot{X}_{E 89} \\
& X_{s d r}=\left[\begin{array}{c}
X \\
Y \\
Z
\end{array}\right] ; T=\left[\begin{array}{c}
T_{x} \\
T_{y} \\
T_{z}
\end{array}\right]=\left[\begin{array}{c}
0.056 \\
0.048 \\
-0.037
\end{array}\right] \mathrm{m} ; \\
& \dot{R}=\left[\begin{array}{ccc}
0 & -\dot{R}_{z} & \dot{R}_{y} \\
\dot{R}_{z} & 0 & -\dot{R}_{x} \\
-\dot{R}_{y} & \dot{R}_{x} & 0
\end{array}\right]= \\
&=\left[\begin{array}{ccc}
0 & 0.781 & 0.518 \\
-0.781 & 0 & -0.054 \\
-0.518 & 0.054 & 0
\end{array}\right] \text { mas } / y
\end{aligned}
$$

where $X$ represents the coordinates vector, $T$ the translation vector and $\dot{R}$ the rotation matrix, respectively.

The second is the well known Helmert transformation (Watson, 2006; Capra and Costantino, 2007) characterised by 7 parameters (three rotations, three translations and one scale factor) which are estimated by means of a least squares process based on a series of points with coordinates known both in IGS05 and
IGM95. It is very important that these points are distributed as homogeneously as possible in the area of interest. The TRASFO v. 1.0 software (Caldera and Visconti, 2006) was used to adjust in the least square sense the 7 parameters from the double sets of coordinates. Below we represent the IGS05-ETRF89 Helmert transformation equation with seven parameters:

$$
\begin{aligned}
& X_{E 89}=T+(1+k) \cdot R \cdot X_{I 05} \\
& X_{s d r}=\left[\begin{array}{l}
X \\
Y \\
Z
\end{array}\right] ; T=\left[\begin{array}{l}
T_{x} \\
T_{y} \\
T_{z}
\end{array}\right] ; k ; R=\left[\begin{array}{ccc}
1 & R_{z} & -R_{y} \\
-R_{z} & 1 & R_{x} \\
R_{y} & -R_{x} & 1
\end{array}\right] ;
\end{aligned}
$$

where $X$ represents the coordinates vector, $T$ the translation vector, $k$ the scale factor and $R$ the rotation matrix, respectively.

Taking into account the data availability the transformation was implemented starting from the Northern Italian area. In this case both methodologies were taken into account and tested. As shown in Section 4.1, of the adopted methods the BA transformation one provides the best results, for this reason we chose this approach. According to this assumption and in the consistency point of view, only the BA type of transformation was tested for the Central and the Southern Italian areas.

With respect to the Boucher-Altamimi approach, two main options are investigated depending on the mean velocity assigned to the area.

In the first a mean velocity is obtained by means of the weighted average of the ETRS89 velocities published for the IGS permanent stations (PSs) located all around the area of interest. We adopted as weight for the velocity the inverse of the distance of each IGS PS chosen for the estimation with respect to their barycentre.

In the second, the velocity is obtained thanks to the weighted average of the ETRS89 velocities (Boucher and Altamimi, 1992) published for the IGS permanent stations (PSs) located all around the area of interest. The weight for velocities depends on the inverse of the distance for each IGS PS with respect to the barycentre of the ItalPoS PSs distributed in the area of interest.

The parameters were chosen by taking into account the most effective residuals minimization, following this philosophy: for each area, all 
Table II. Results of the comparison between the IGS05 coordinates of reference sites computed by the EUREF analysis centres with the Bernese $5.0 \mathrm{sw}$ (epoch 2007.795) and those estimated in our solution by means of the Globk sw (epoch 2007.860). Rows in bold type underline residuals of the Italian GNSS sites.

\begin{tabular}{cccccccc}
\hline \hline$P S$ & $\Delta X[\mathrm{~cm}]$ & $\Delta Y[\mathrm{~cm}]$ & $\Delta Z[\mathrm{~cm}]$ & $P S$ & $\Delta X[\mathrm{~cm}]$ & $\Delta Y[\mathrm{~cm}]$ & $\Delta Z[\mathrm{~cm}]$ \\
\hline VILL & 0.60 & 0.00 & 0.50 & ZOUF & $\mathbf{- 0 . 2 0}$ & $\mathbf{0 . 1 0}$ & $\mathbf{0 . 2 0}$ \\
SFER & 0.30 & 0.00 & 0.30 & WTZR & -0.30 & 0.10 & -0.20 \\
RABT & 0.30 & 0.10 & 0.40 & ONSA & -0.40 & 0.10 & -0.10 \\
HOFN & -0.10 & -0.20 & -0.10 & NYA1 & -0.10 & -0.20 & 0.40 \\
REYK & 0.00 & -0.30 & 0.50 & PRAT & $\mathbf{- 0 . 1 0}$ & $\mathbf{0 . 1 0}$ & $\mathbf{0 . 2 0}$ \\
TRAB & -0.70 & -0.10 & -0.10 & ELBA & $\mathbf{- 0 . 1 0}$ & $\mathbf{0 . 2 0}$ & $\mathbf{0 . 0 0}$ \\
RAMO & -0.70 & -0.30 & -0.30 & COMO & $\mathbf{- 0 . 2 0}$ & $\mathbf{0 . 0 0}$ & $\mathbf{0 . 1 0}$ \\
GLSV & -0.60 & -0.30 & -0.30 & CAGL & $\mathbf{- 0 . 1 0}$ & $\mathbf{0 . 0 0}$ & $\mathbf{- 0 . 1 0}$ \\
METS & -0.40 & -0.10 & -0.30 & ZIMM & -0.30 & 0.00 & -0.10 \\
JOZE & -0.10 & 0.00 & 0.30 & GRAS & -0.10 & 0.00 & 0.20 \\
TRO1 & 0.80 & 0.20 & 2.90 & WSRT & -0.30 & 0.10 & 0.10 \\
BOR1 & -0.10 & 0.20 & 0.20 & BRUS & -0.10 & 0.10 & 0.20 \\
MATE & $\mathbf{- 0 . 5 0}$ & $\mathbf{0 . 0 0}$ & $\mathbf{- 0 . 3 0}$ & MOSE & $\mathbf{- 0 . 1 0}$ & $\mathbf{0 . 2 0}$ & $\mathbf{0 . 0 0}$ \\
GRAZ & -0.30 & 0.00 & -0.10 & MOPS & $\mathbf{0 . 0 0}$ & $\mathbf{0 . 1 0}$ & $\mathbf{0 . 0 0}$ \\
NOT1 & $\mathbf{- 0 . 1 0}$ & $\mathbf{0 . 1 0}$ & $\mathbf{0 . 0 0}$ & ROVE & $\mathbf{- 0 . 2 0}$ & $\mathbf{0 . 1 0}$ & $\mathbf{0 . 0 0}$ \\
POTS & -0.40 & 0.00 & -0.20 & TORI & $\mathbf{- 0 . 2 0}$ & $\mathbf{0 . 1 0}$ & $\mathbf{- 0 . 1 0}$ \\
\hline
\end{tabular}

the estimated parameters sets were applied to a series of test surveys carried out almost in all the Italian Regions. The purpose was to check the parameters reliability and to suggest the best ones for the final transformation, to transform site coordinates from the IGS05 reference frame to the national one (the IGM95-ETRF89). The test surveys, carried out by means of several GPS receivers, had to simulate the customer's behaviour during his work, like for example the positioning of any unknown point. At the same time two more GPS receivers were tracking being placed on two benchmarks belonging to the IGM95 Italian geodetic network. These benchmarks are fixed points, with known coordinates both in horizontal position and height. At the end of the survey, the first step was the post-processing of the unknown point with respect to the IGM95 benchmarks constrained using the values of the coordinates provided by Istituto Geografico Militare Italiano (IGMI). At this stage the IGM95-ETRF89 coordinates of the point of interest were estimated; these were used as reference values to test the quality of the transformation parameters. In a second step, the postprocessing of the unknown point was performed with respect to some ItalPoS PSs (no more than two or three) located near the point and constrained by means of their coordinates, provided by the adjustment with Gamit/Globk (see Section 3). At this stage the IGS05 coordinates of the point of interest were estimated. All the pa- 


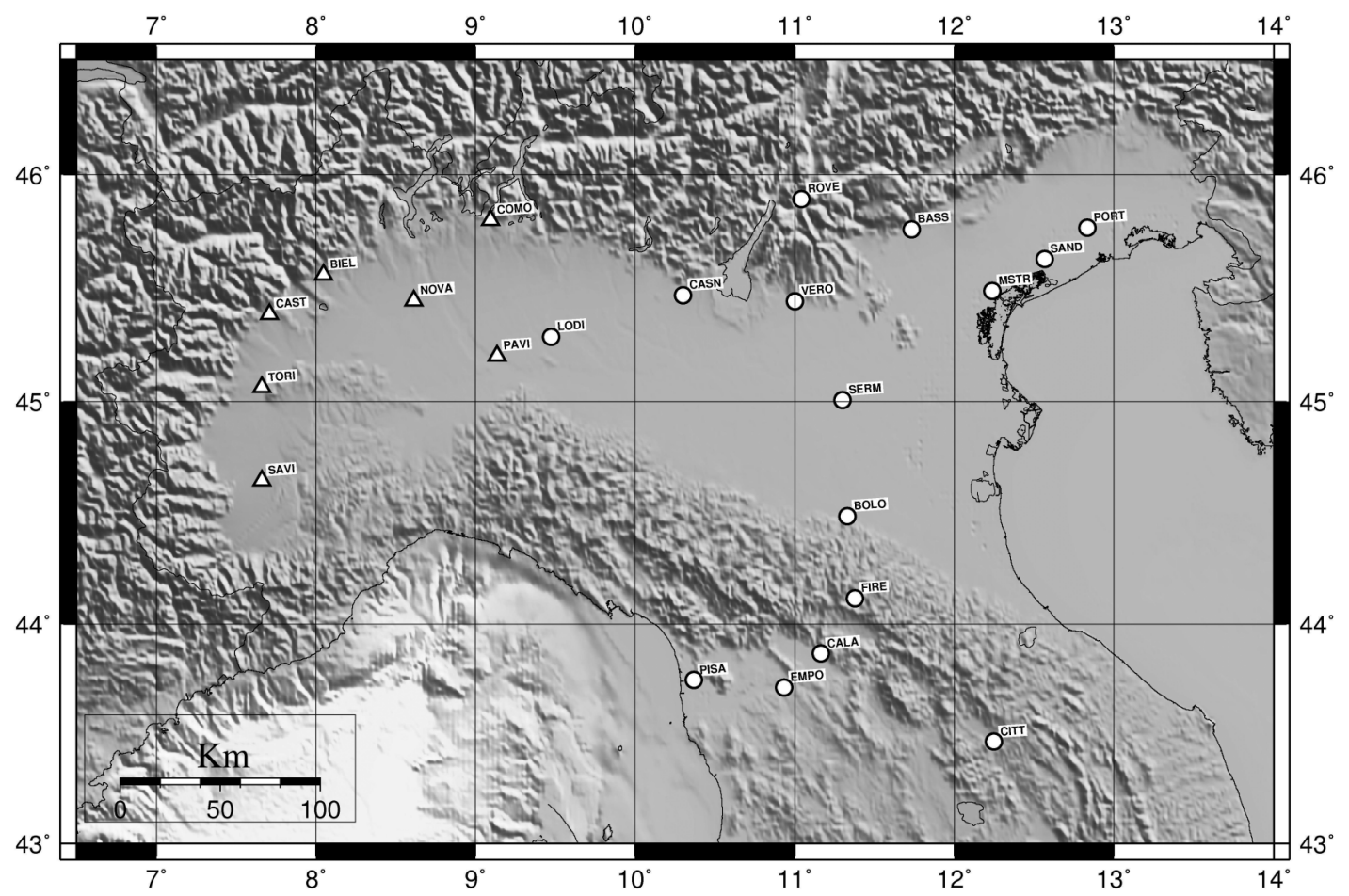

Fig. 2. Northern Italy cluster: points known both in the IGS05 and in the IGM95-ETRF89, used for the transformation tests. Circular icons: points for the North-East transformation. Triangle-shaped icons: points for the North-West transformation.

rameters of transformation estimated were applied to the IGS05 coordinates to obtain the IGM95-ETRF89 transformed coordinates. The last step was the comparison between the IGM95-ETRF89 reference coordinates and the IGM95-ETRF89 coordinates transformed from the IGS05.

The differences in the comparison have to be as small as possible; the purpose is to have residuals lower than the IGM95 network nominal accuracy that is $5 \mathrm{~cm}$ in horizontal position and $10 \mathrm{~cm}$ in height.

\subsection{Estimation of ItalPoS Network Transfor- mation parameters}

Taking into account the data availability, the transformation estimation started from the
Northern cluster (including all the Regions in the North of Italy: Val D'Aosta, Piedmont, Liguria, Lombardy, Veneto, Trentino Alto Adige, Friuli Venezia Giulia, Emilia Romagna). In this case six parameter sets were calculated, applied to the IGS05 coordinates and finally compared with the IGM95-ETRF89 reference coordinates to reach the best final solution. Both the methodologies, the BA and the Helmert transformation, were investigated.

Concerning the Helmert transformation, many points with coordinates known both in the IGS05 and in the IGM95-ETRF89 were available. Some of them come from the Politecnico of Torino GNSS permanent network and others were provided by Leica Geosystems (fig. 2). At first sight the North area seemed very large so it was divided into two: the North-East where the Leica Geosystems points were used 
for the transformation estimation (there were 15 points available for this area: 8 were used to find out transformation parameters while the other 7 as control points) and the North-West where the Politecnico of Torino points were used (in this case only 7 points were available so that 4 were used to estimate parameters and 3 to check results). At this step two parameter sets were available with the Helmert approach, according to the area dimension and the points distribution: the North-East and the North-West ones respectively.

Concerning the BA type of transformation, a further four sets were calculated depending on the mean velocity attributed to the area:

- ETRS89 velocity published for the IGS PS of Padua (PADO);

- ETRS89 velocity published for the IGS PS of Turin (IENG);

- Velocity calculated by a weighted average of the ETRS89 velocities published for a set of IGS PSs distributed in the Northern area (PADO, GRAS, GRAZ, ZIMJ, GENO, IENG, HFLK). Each of these velocities was weighted using the inverse distance of the specific IGS PS with respect to their barycentre.

- Velocity calculated by a weighted average of the ETRS89 velocities published for the IGS PSs distributed in the Northern area (PADO, GRAS, GRAZ, ZIMJ, GENO, IENG, HFLK). Each of these velocities was weighted by the inverse distance of the specific IGS PS with respect to the barycentre of all the ItalPoS PSs included in the Northern Italian area (the PSs taken into account were only those operating at the time of the first adjustment, September 2007).

The transformation parameters were estimated according to the approaches described above. In the meantime some surveys, at least three hours long, have been carried out in nearly every Region of the Northern area. The comparison results are shown in table III together with the detailed residuals statistic of each parameters set investigated. This makes the comparison easier and allows the best final solution to be obtained for the Northern area.

Analysing the residuals in table III the Helmert solutions seems worse. Indeed, although mean values are lower than the accuracy provided from the IGM95 static network, their standard deviations are the highest and even maximun and minimum values are too much large if compared with the accuracy expected and with the performances of other solutions. Accordingly, the methodology chosen was the Boucher-Altamimi approach. It is important and necessary to take into account that another key reason in decision-making is the logistic aspect. The Helmert approach requires GPS campaigns all over Italy focused to find enough points with known coordinates both in IGS05 and IGM95-ETRF89.

The more points are available, the better the parameters estimated will be but this requires time to cover even Central and Southern Italy and, not less important, people able to survey points following all the guidelines to obtain high accuracy. Taking into account the long time to obtain data without definite reliability and more time to find some control points, the best decision both in terms of precision and time optimization is the Boucher-Altamimi approach. The best solution among the possible options is the velocity weighted with respect to the ItalPoS PSs barycentre: indeed the mean values are lower than the IGM95 accuracy both in horizontal position and in height. We found only few outliers in height, as shown in the minimum indicator which is slightly greater than the accuracy required, $10 \mathrm{~cm}$. This is mainly due to the fact that the ETRF89 coordinates used as the reference ones were calculated about twenty years ago. It is necessary to take into account that during this long period some IGM95 benchmarks could have been moved not only because of natural reasons (crustal deformation, tectonic movements) but also by human actions. A Boucher-Altamimi with a velocity area based on the ItalPoS PSs barycentre is the final type of transformation applied and the same strategy was also followed for the other areas.

The procedure previously described was also applied to the Central (including all the Regions in the Centre of Italy: Tuscany, Umbria, Marche, Abruzzo, Lazio, Molise) and Southern (including all the Regions in the South of Italy: Campania, Puglia, Calabria, Basilicata, Sicilia, Sardegna) Italian clusters of the ItalPoS network and the results are shown in tables IV and V. Having 
Table III. Northern Italy cluster: statistical summary of the transformation parameter sets estimated. For each one residuals of the comparison between reference coordinates and transformed coordinates in ETRF89 are reported in geographic coordinates in order to simplify discussion and conclusions.

\begin{tabular}{lcccccc}
\hline \hline \multirow{2}{*}{ Type of transformation } & \multicolumn{2}{c}{ Mean [cm] } & \multicolumn{3}{c}{ Max [cm] } \\
\cline { 2 - 7 } & $\Delta \varphi$ & $\Delta \lambda$ & $\Delta h \_$ell & $\Delta \varphi$ & $\Delta \lambda$ & $\Delta h \_$ell \\
\cline { 2 - 7 } ETRF89 (BA PADO) & 0.32 & 0.04 & 1.64 & 4.50 & 4.40 & 11.90 \\
ETRF89 (BA IENG) & 4.56 & -0.42 & -4.48 & 8.60 & 3.40 & 5.90 \\
ETRF89 (BA IGS barycentre) & 2.62 & -0.54 & -1.96 & 6.80 & 3.50 & 8.30 \\
ETRF89 (BA ItalPoS barycentre) & 2.90 & -0.54 & -1.98 & 7.00 & 3.40 & 8.30 \\
ETRF89 (North-West) & -1.36 & -4.30 & -7.62 & 2.20 & 6.70 & 2.10 \\
ETRF89 (North-East) & -0.02 & 1.68 & 0.86 & 3.00 & 10.40 & 13.80 \\
\hline & & Min [cm] & & $\sigma[\mathbf{c m}]$ & \\
Type of transformation & $\Delta \varphi$ & $\Delta \lambda$ & $\Delta h \_e l l$ & $\Delta \varphi$ & $\Delta \lambda$ & $\Delta h \_e l l$ \\
\hline ETRF89 (BA PADO) & -2.10 & -3.40 & -9.90 & 2.45 & 2.88 & 8.93 \\
ETRF89 (BA IENG) & 2.30 & -3.90 & -16.00 & 2.37 & 2.80 & 8.96 \\
ETRF89 (BA IGS barycentre) & 0.30 & -4.00 & -13.50 & 2.43 & 2.83 & 8.96 \\
ETRF89 (BA ItalPoS barycentre) & 0.50 & -3.90 & -13.50 & 2.40 & 2.75 & 8.95 \\
ETRF89 (North-West) & -7.00 & -12.30 & -19.90 & 3.24 & 6.35 & 9.28 \\
ETRF89 (North-East) & -3.60 & -2.90 & -11.10 & 2.34 & 4.67 & 8.76 \\
\hline
\end{tabular}

fixed the Boucher-Altamimi type of transformation, several velocity options were taken into account, just to be sure that the ItalPoS barycentre solution was the best one. Central and Southern Italy have a very low number of IGS sites, so the choice is very limited. Nevertheless the best and most uniform distribution all around the cluster was chosen also depending on the data availability. Concerning the Central cluster, there are two configurations for the IGS PSs taken into account to find the mean velocity for the area: GENO, CAGL, MATE and SOFI or GENO, CAGL and MATE. In the North direction MEDI was not working so that GENO was chosen. The use of SOFI for the East direction was investigated. Both the weighting strategy with respect to the IGS PSs barycentre or the ItalPoS PSs were tested. Residual statistic indicators are shown in table IV.

Concerning the Southern cluster, the IGS PSs taken into account to find the mean velocity for the area are: GENO, CAGL, MATE, NOT1 and LAMP. Also in this case MEDI rinex data were not available. Both the weighting strategies with respect to the IGS PSs barycentre or the ItalPoS PSs were tested and the residual statistic indicators are shown in table V.

Even in Central and Southern areas the best solution was the Boucher-Altamimi transformation with the velocities weighted adopting the ItalPoS PSs barycentre strategy previously described. The mean residuals were always lower than the IGM95 accuracy so that transformation parameters are successful. Table VI reports the 
Table IV Central Italy cluster: statistical summary of the transformation parameter sets estimated. For each one residuals of the comparison between reference coordinates and transformed coordinates in ETRF89 are reported in geographic coordinates in order to simplify discussion and conclusions.

\begin{tabular}{|c|c|c|c|c|c|c|}
\hline \multirow{2}{*}{ Type of transformation } & \multicolumn{3}{|c|}{ Mean [cm] } & \multicolumn{3}{|c|}{$\operatorname{Max}[\mathrm{cm}]$} \\
\hline & $\Delta \varphi$ & $\Delta \lambda$ & $\Delta h \_e l l$ & $\Delta \varphi$ & $\Delta \lambda$ & $\Delta h \_e l l$ \\
\hline ETRF89 (BA IGS barycentre+SOFI) & 2.20 & 0.11 & -8.63 & 5.37 & 6.48 & -3.28 \\
\hline ETRF89 (BA IGS barycentre) & 2.82 & 0.46 & -8.21 & 5.88 & 6.81 & -2.92 \\
\hline ETRF89 (BA ItalPoS barycentre+SOFI) & 3.53 & 0.14 & -8.32 & 6.66 & 6.60 & -3.01 \\
\hline ETRF89 (BA ItalPoS barycentre) & 2.58 & 0.30 & -8.30 & 5.67 & 6.78 & -2.98 \\
\hline \multirow{2}{*}{ Type of transformation } & \multicolumn{3}{|c|}{$\operatorname{Min}[\mathrm{cm}]$} & \multicolumn{3}{|c|}{$\sigma[\mathrm{cm}]$} \\
\hline & $\Delta \varphi$ & $\Delta \lambda$ & $\Delta h \_e l l$ & $\Delta \varphi$ & $\Delta \lambda$ & $\Delta h \_e l l$ \\
\hline ETRF89 (BA IGS barycentre+SOFI) & -2.13 & -5.76 & -15.45 & 2.39 & 4.17 & 3.71 \\
\hline ETRF89 (BA IGS barycentre) & -1.47 & -5.34 & -15.01 & 2.37 & 4.18 & 3.70 \\
\hline ETRF89 (BA ItalPoS barycentre+SOFI) & -0.78 & -5.73 & -15.13 & 2.38 & 4.21 & 3.72 \\
\hline ETRF89 (BA ItalPoS barycentre) & -1.71 & -5.52 & -15.16 & 2.37 & 4.18 & 3.72 \\
\hline
\end{tabular}

Table V. Southern Italy cluster: statistical summary of the transformation parameter sets estimated. For each one residuals of the comparison between reference coordinates and transformed coordinates in ETRF89 are reported in geographic coordinates in order to simplify discussion and conclusions.

\begin{tabular}{|c|c|c|c|c|c|c|}
\hline \multirow{2}{*}{ Type of transformation } & \multicolumn{3}{|c|}{ Mean [cm] } & \multicolumn{3}{|c|}{$\operatorname{Max}[\mathrm{cm}]$} \\
\hline & $\Delta \varphi$ & $\Delta \lambda$ & $\Delta h \_e l l$ & $\Delta \varphi$ & $\Delta \lambda$ & $\Delta h \_e l l$ \\
\hline ETRF89 (BA IGS barycentre) & -0.50 & 4.41 & -3.48 & 5.67 & 9.42 & 6.28 \\
\hline ETRF89 (BA ItalPoS barycentre) & -1.52 & 4.63 & -3.48 & 4.68 & 9.57 & 6.34 \\
\hline \multirow{2}{*}{ Type of transformation } & \multicolumn{3}{|c|}{$\operatorname{Min}[\mathrm{cm}]$} & \multicolumn{3}{|c|}{$\sigma[\mathrm{cm}]$} \\
\hline & $\Delta \varphi$ & $\Delta \lambda$ & $\Delta h \_e l l$ & $\Delta \varphi$ & $\Delta \lambda$ & $\Delta h \_e l l$ \\
\hline ETRF89 (BA IGS barycentre) & -6.33 & -1.77 & -13.15 & 3.49 & 3.40 & 7.76 \\
\hline ETRF89 (BA ItalPoS barycentre) & -7.32 & -1.47 & -13.16 & 3.49 & 3.37 & 7.77 \\
\hline
\end{tabular}

final parameters (three translations and three rotations) for the IGS05-ETRF89 transformation, valid for coordinates conversion in the three clusters composing the whole ItalPoS GNSS permanent network territory.

Particular attention should be reserved to the transition areas of the network where two differ- ent transformation parameters sets can be applied to provide coordinates conversion from the IGS05 to the ETRF89 reference frames. The interested Regions are Emilia Romagna, Tuscany, Marche between the Northern and the Central transformation sets and Lazio, Molise, Campania between the Central and the Southern ones. 
Table VI. Final parameters of the IGS05-ETRF89 transformation for the clusters in which ItalPoS network was divided.

\begin{tabular}{lcccccc}
\hline \hline $\begin{array}{l}\text { Transformation } \\
\text { parameters }\end{array}$ & $t_{x}[\mathrm{~m}]$ & $t_{y}[\mathrm{~m}]$ & $t_{z}[\mathrm{~m}]$ & $r_{x}[\mathrm{rad}]$ & $r_{y}[\mathrm{rad}]$ & $r_{z}[\mathrm{rad}]$ \\
\hline Northern & 0.05901380 & 0.03839360 & -0.05564760 & $4.93 \mathrm{E}-09$ & $4.73 \mathrm{E}-08$ & $-7.13 \mathrm{E}-08$ \\
Central & 0.07225924 & 0.04156468 & -0.06970175 & $4.93 \mathrm{E}-09$ & $4.73 \mathrm{E}-08$ & $-7.13 \mathrm{E}-08$ \\
Southern & 0.08864580 & 0.07103398 & -0.08996419 & $4.93 \mathrm{E}-09$ & $4.73 \mathrm{E}-08$ & $-7.13 \mathrm{E}-08$ \\
\hline
\end{tabular}

Table VII.Transformation performances in the transition areas: some points were surveyed to check if results are the same applying both parameters sets estimated for the neighbouring clusters.

\begin{tabular}{lcccccc}
\hline \hline \multirow{2}{*}{$\begin{array}{l}\text { Check points } \\
\text { in transition area }\end{array}$} & \multicolumn{2}{c}{ Residuals with Northern parameters } & \multicolumn{3}{c}{ Residuals with Central parameters } \\
\cline { 2 - 6 } Marina Pietrasanta & 4.14 & -3.93 & -11.91 & 1.95 & -3.75 & -11.9 \\
Pistoia & 7.89 & -3.30 & -15.16 & 5.61 & -3.3 & -15.16 \\
Scansano & 0.51 & 0.39 & -8.73 & 0.51 & -5.52 & -4.38 \\
Cortona & 5.43 & -4.29 & -7.24 & 3.27 & -4.17 & -7.21 \\
Ancona & 7.92 & 0.12 & -6.91 & 5.67 & 0.15 & -6.81 \\
Castelfranco E. & 7.02 & -2.46 & -13.52 & 4.8 & -2.34 & -13.47 \\
\hline Check points & Residuals with Northern parameters & Residuals with Central parameters \\
\cline { 2 - 7 } in transition area & $\Delta \varphi[\mathrm{cm}]$ & $\Delta \lambda[\mathrm{cm}]$ & $\Delta h \_e l l[\mathrm{~cm}]$ & $\Delta \varphi[\mathrm{cm}]$ & $\Delta \lambda[\mathrm{cm}]$ & $\Delta h \_e l l[\mathrm{~cm}]$ \\
\hline Campobasso & 3.6 & 6.78 & -2.98 & 0.66 & 9.90 & -2.62 \\
Roma & 2.61 & 5.28 & -8.76 & -0.33 & 8.70 & -8.52 \\
Avellino & 3.87 & 6.39 & 1.87 & 0.90 & 9.57 & 2.31 \\
\hline
\end{tabular}

The unknown position should always be the same within the range of accuracy; this implies that results cannot be different. To check the congruity of this hypothesis some check points with known ETRF89 coordinates were surveyed and then adjusted constraining the ItalPoS PSs with their IGS05 coordinates. The two possible transformation parameters were applied and residuals between them and the reference coordinates calculated. Table VII shows these residuals so that it is possible to make a comparison: most residuals are similar adopting both the options and even where some difference exists it is in any case within the IGM95 nominal accuracy.

A further comparison was made between the ETRF89 coordinates of the ItalPoS PSs estimated following the procedure previously described by means of the Boucher-Altamimi transformation, and those obtained by applying the GLORG (GLobal ORiGin) module of the Globk software to stabilise the network coordinates in the ETRF2000 at epoch 1989.0 using a set of velocities and coordinates taken from EUREF facility combined solutions. The results of this comparison are shown in table VIII; they are well below the nominal accuracy of 
Table VIII. Residuals between ETRF89 cartesian coordinates estimated by means of the Globk stabilisation process and BA transformation.

\begin{tabular}{|c|c|c|c|c|c|c|c|}
\hline$P S$ & $\Delta X[\mathrm{~cm}]$ & $\Delta Y[\mathrm{~cm}]$ & $\Delta Z[\mathrm{~cm}]$ & $P S$ & $\Delta X[\mathrm{~cm}]$ & $\Delta Y[\mathrm{~cm}]$ & $\Delta Z[\mathrm{~cm}]$ \\
\hline GRAV & 4.86 & 1.18 & 0.34 & GAZZ & 4.73 & 1.57 & 0.17 \\
\hline TORI & 4.82 & 1.34 & 0.38 & PALA & 5.02 & 1.24 & 0.34 \\
\hline CAST & 5.00 & 1.22 & 0.38 & CASN & 3.74 & 1.55 & 1.89 \\
\hline SAVI & 5.35 & 1.09 & 0.23 & PARM & 4.93 & 1.11 & 0.18 \\
\hline CUNE & 8.72 & 3.01 & -3.47 & CARP & 1.43 & -0.68 & 4.23 \\
\hline MOND & 4.08 & 1.59 & 0.12 & SIRM & 4.98 & 1.20 & 0.21 \\
\hline BIEL & 4.58 & 1.19 & 0.78 & MAGA & 5.14 & 1.21 & 0.31 \\
\hline CRES & 4.90 & 1.23 & 0.37 & SBPO & 4.97 & 1.18 & 0.25 \\
\hline ASTI & 5.25 & 0.99 & 0.07 & VERO & 4.97 & 1.24 & 0.15 \\
\hline NOVA & 5.03 & 1.40 & 0.18 & MOD1 & 6.76 & 1.87 & -1.81 \\
\hline ALES & 4.96 & -0.03 & 1.89 & MODE & 4.97 & 1.12 & 0.19 \\
\hline COMO & 4.94 & 1.29 & 0.36 & ROVE & 6.29 & 1.23 & -0.78 \\
\hline PAVI & 5.39 & 1.83 & -0.21 & SERM & 4.94 & 1.22 & 0.15 \\
\hline VARZ & 4.90 & 1.14 & 0.26 & BASS & 5.07 & 1.19 & 0.20 \\
\hline STUE & 5.01 & 1.24 & 0.21 & BOLO & 4.85 & 1.10 & 0.04 \\
\hline COLI & 3.87 & 0.84 & 0.93 & VENE & 5.01 & 1.23 & 0.12 \\
\hline MORB & 4.78 & 1.36 & 0.41 & SAND & 5.02 & 1.18 & 0.14 \\
\hline LODI & 4.67 & 1.45 & 0.31 & PORT & 4.96 & 1.19 & 0.10 \\
\hline PIAC & 4.90 & 1.23 & 0.25 & BRAS & 4.97 & 1.14 & 0.17 \\
\hline BRUG & 4.93 & 1.15 & 0.20 & FIRE & 4.98 & 1.15 & 0.10 \\
\hline LASP & 4.97 & 1.11 & 0.20 & & & & \\
\hline
\end{tabular}

$5 \mathrm{~cm}$ in horizontal position and $10 \mathrm{~cm}$ in height.

\section{Discussion and conclusions}

The importance of GNSS permanent networks is growing rapidly and many countries already have developed or are developing their own national networks. The most visible and immediate advantage is for people working on topography and geodesy in terms of reduction of time and money but also for researchers. In addition, a major role of a GNSS permanent network is the materialization of national reference frames in a new point of view, dynamic in- 
stead of static by means of continuous data processing. ItalPoS, Italian Positioning Service by Leica Geosystems Italian Division, is a new private GNSS permanent network uniformly covering the whole Italian peninsula.

Thanks to more than 130 permanent stations, ItalPoS provides users both with RINEX data for post-processing and real-time area corrections.

The entire network has been constrained to some EUREF permanent stations, processed and adjusted in the IGS05 reference frame by means of software Gamit/Globk. To provide users with coordinates in the official Italian reference frame, IGM95-ETRF89, transformation parameters sets have been estimated for three clusters composing the ItalPoS network to perform the coordinates conversion. Several parameters were estimated by means of two main approaches: the Helmert with seven parameters and the Boucher-Altamimi transformations. Comparisons highlight the best results for the Boucher-Altamimi strategy whose residuals are lower than the IGM95 nominal accuracy. The congruity in transition areas was thoroughly verified and a further comparison was made between the parameters estimated and those from the Glorg (GLobal ORiGin) module of the Globk software; also in this case residuals are lower than the accuracy target. As a consequence, due to the procedure accuracy, the final solutions and all transformation parameters here presented could be available for private users and the scientific community.

\section{Acknowledgements}

We are grateful to all the operators maintaining the permanent stations belonging to the Institutions involved on ItalPoS permanent service in particular: IGS, INGV, ASI, EUREF and Leica Geosystems GPS division.

We are grateful to Leica Geosystems GPS division for the data availability. We thank Prof. P. Baldi whose review improved the manuscript.

The figures of this paper were produced by means of Generic Mapping Tools (GMT) graphic package (Wessel and Smith, 1998).

\section{REFERENCES}

Altamimi, Z., X. Collilieux, J. Legrand, B. Garayt and C. BOUCHER (2007): ITRF2005: A new release of the International Terrestrial Reference Frame based on time series of station positions and Earth Orientation Parameters, J. Geophys. Res., 112, B09401, doi:10.1029/2007JB004949.

Biagi, L., S. Caldera, A. Capra, C. Castagnetti and F. SANSÒ (2008): Densification of IGS/EPN by local permanent networks: sensitivity of results with respect to the adjustment choices, Bulletin of Geodesy and Geomatics, peer review completed and paper accepted by reviewers.

Boucher, C. and Z. Altamimi (1992): The EUREF Terrestrial Reference System and its first realizations, (EUREF Meeting, Bern, Switzerland March 4-6, 1992).

Boucher, C. and Z. Altamimi (2007): Memo: Specifications for reference frame fixing in the analysis of a EUREF GPS campaign, (available on line at http://users.auth.gr/ kvek/20070327-MEMOver6.pdf).

BRUYNINX, C. (2004): The EUREF Permanent Network: a multi-disciplinary network serving surveyors as well as scientists, GeoInformatics, 7, 32-35.

Caldera, S. and M.G. Visconti (2006): Manuale_TRASFO v 1.0, (available on line at http://geomatica.como.polimi.it/software/prin2004.php).

CAPRA, A. and D. Costantino (2007): GEOMATICA per corsi di laurea e di laurea specialistica (magistrale), (Mandese Editore, Taranto, Italy), chapters 4 and 8.

Casula, G., M. Dubbini and A. Galeandro (2007): Modeling environmental bias and computing velocity field from data of Terra Nova Bay network in Antartica by means of a quasi-observation processing approach, U.S. Geological Survey and the National Academies, (Short research paper), USGS OF-2007-1041, doi:10.3133/of2007-1047.srp054.

Dong, D., T.A. HERring and R.W. KING (1998): Estimating regional deformation from a combination of space and terrestrial geodetic data, J Geodesy, 72, 200-214.

KenYeRES, A. and C. BRUYNINX (2004): Monitoring of the EPN Coordinate Time Series for Improved Reference Frame Maintenance, GPS solutions, 8 (4), pp. 200-209.

Herring, T.A., R.W. KING and S.C. MCCLUSKY (2006a): GPS Analysis at MIT, GAMIT Reference Manual, Release 10.3, (Department of Earth, Atmospheric, and Planetary Sciences Massachusetts Institute of Technology, Cambridge MA, available on line at http://chandler.mit.edu/ simon/gtgk/GAMIT_Ref_10.3.pdf, accessed 14 May 2008).

Herring, T.A., R.W. KING and S.C. MCClusky (2006b): Global Kalman filter VLBI and GPS analysis program, GLOBK Reference Manual, Release 10.3, (Department of Earth, Atmospheric, and Planetary Sciences Massachusetts Institute of Technology, Cambridge MA, available on line at http://chandler.mit.edu/ simon/gtgk/GLOBK_Ref_10.3.pdf, accessed 23 May 2008).

LEICA GEOSYSTEMS (2005): White Paper: GPS SpiderNET - Take it to the MAX, (available on line at http://www.leica-geosystems.com/corporate/en/downloads/lgs_page_catalog.htm?cid=3159, accessed 18 February 2009). 
SElvagGI, G. and RING WORKING GROUP (2006): La Rete Integrata Nazionale GPS (RING) dell' INGV: una infrastruttura aperta per la ricerca scientifica, $I N G V$ Technical Report, p. 1-6.

Serpelloni, E., G. Casula, A. Galvani, M. Anzidei and P. BALDI (2006): Data analysis of permanent GPS networks in Italy and surrounding regions: application of a distributed processing approach, Ann Geophys, 49 (4/5), 897-928.

SOPAC (2008): SOPAC Facilities site, (available on line at http://sopac.ucsd.edu/other/facilities.html, accessed 20 May 2008).
Watson, G.A. (2006): Computing Helmert transformations, Journal of Computational and Applied Mathematics, 197, 387-395.

Wessel, P. and W.H.F. SMith (1998): New improved version of Generic Mapping Tools Released, EOS Trans. $A G U, 79$ (47), 579.

(received March 23, 2009;

accepted April 29, 2009) 